\title{
Lunar cratering chronology: Statistical fluctuation of crater production frequency and its effect on age determination
}

\author{
Tomokatsu Morota $^{1}$, Jun'ichi Haruyama ${ }^{1}$, Chikatoshi Honda ${ }^{1}$, Yasuhiro Yokota ${ }^{1}$, \\ Makiko Ohtake $^{1}$, Yoshiko Ogawa ${ }^{2}$, and Tsuneo Matsunaga ${ }^{2}$ \\ ${ }^{1}$ Institute of Space \& Astronautical Science, Japan Aerospace Exploration Agency, 3-1-1 Yoshinodai, Sagamihara 229-8510, Japan
${ }^{2}$ Center for Global Environmental Research, National Institute for Environmental Studies, 16-2 Onogawa, Tsukuba 305-8506, Japan
}

(Received March 30, 2007; Revised August 9, 2007; Accepted October 31, 2007; Online published April 9, 2008)

\begin{abstract}
The crater frequency is often used to determine ages of planetary surfaces. In this study, we evaluate the statistical fluctuation of the crater frequency and its effect on age determination using a Monte Carlo simulation prior to the SELENE (KAGUYA) / Terrain Camera acquiring extensive high-resolution images over the entire Moon. We found that the ages estimated between 2.5 and 3.5 Gyr tend to be too young when there are not enough craters. Age determination for Eratosthenian units is least accurate. The errors defined with $1 \sigma$ are 0.7 Gyr for units of $100 \mathrm{~km}^{2}, 0.5 \mathrm{Gyr}$ for units of $1000 \mathrm{~km}^{2}$, and $0.4 \mathrm{Gyr}$ for units of $2500 \mathrm{~km}^{2}$. The maximum error exceeds $20 \%$ and is comparable with the error due to the cratering asymmetry.
\end{abstract}

Key words: Moon, cratering chronology, crater size-frequency distribution, statistical fluctuation.

\section{Introduction}

Dating of geological units of planetary surfaces is one of the most important subjects of planetary science for understanding a planet's origin and evolution. Based on the simple idea that older surfaces accumulate more craters, we can infer relative ages by measuring the crater frequencies with remote-sensing image data. The lunar cratering chronology formulated by relating crater frequencies to the radiometric ages of Apollo and Luna samples (Hartmann, 1970, 1972; Neukum et al., 1975b; Neukum, 1983; Neukum and Ivanov, 1994) enables us to convert the crater frequencies into absolute ages. The method of dating by crater counting has been widely used to infer the lunar geological history (e.g., Neukum and Konig, 1976; Baldwin, 1985; Wilhelms, 1987; Greeley et al., 1993; Hiesinger et al., 2000, 2003, 2006).

Japanese lunar explorer SELENE (KAGUYA) has been launched in 2007. The Terrain Camera (TC) installed on SELENE will take images of the surface of the entire Moon with a nominal spatial resolution of $10 \mathrm{~m} /$ pixel (Haruyama et al., 2000, 2003, 2006a, 2008; Ohtake et al., 2000). The large numbers of high-resolution images will be used to determine the relative and absolute ages of geological units of the entire Moon. Therefore, it is necessary to discuss the error of the dating technique before the new image data are acquired.

Since a greater number of counted craters is statistically expected to yield better accuracy of age determination, smaller craters must be counted. In contrast, the application of small craters to age determination is problematic because the size-frequency distribution (SFD) of small craters $(<4 \mathrm{~km}$ in diameter) may be complicated by con-

Copyright (C) The Society of Geomagnetism and Earth, Planetary and Space Sciences (SGEPSS); The Seismological Society of Japan; The Volcanological Society of Japan; The Geodetic Society of Japan; The Japanese Society for Planetary Sciences; TERRAPUB tamination of secondary craters (e.g., Namiki and Honda, 2003; McEwen et al., 2005; McEwen and Bierhaus, 2006). The contribution of secondary craters to the population of small craters has been widely discussed based on the lunar and Martian cratering records (e.g., Hirata and Nakamura, 2006; Ivanov, 2006; Dundas and McEwen, 2006) and must continue to be studied in order to assess the reliability of age determination by small craters.

In parallel, it is necessary to investigate the limitation in accuracy of the dating method for considering the significance of future high-resolution images, when we postulate that small craters can be counted for age determination. The main factor limiting the accuracy is statistical fluctuation of the crater production frequency. In this paper, we evaluate the error in age determination due to statistical fluctuation of crater frequency by a numerical Monte Carlo simulation.

\section{Numerical Simulation}

We simulate random sampling of craters from a crater population. The SFD of the sampled craters is measured and converted to the absolute age using the lunar cratering chronology. Iterating this procedure, we evaluate the statistical fluctuation of the crater SFDs and the error of age determination due to the fluctuation. The detailed procedure is presented below.

\subsection{Random sampling of craters}

We assume a crater population with the following simple power-law SFD,

$$
N(D) \propto D^{b} .
$$

Here, $D$ is the crater diameter and $N(D)$ is the number of craters larger than $D$. It is well known that the SFD of lunar mare craters does not follow the simple power-law relation over a wide size range (e.g., Chapman and Haefner, 1967; Neukum et al., 1975a; Namiki and Honda, 2003) but can be approximated by Eq. (1) in a limited size range. In this 

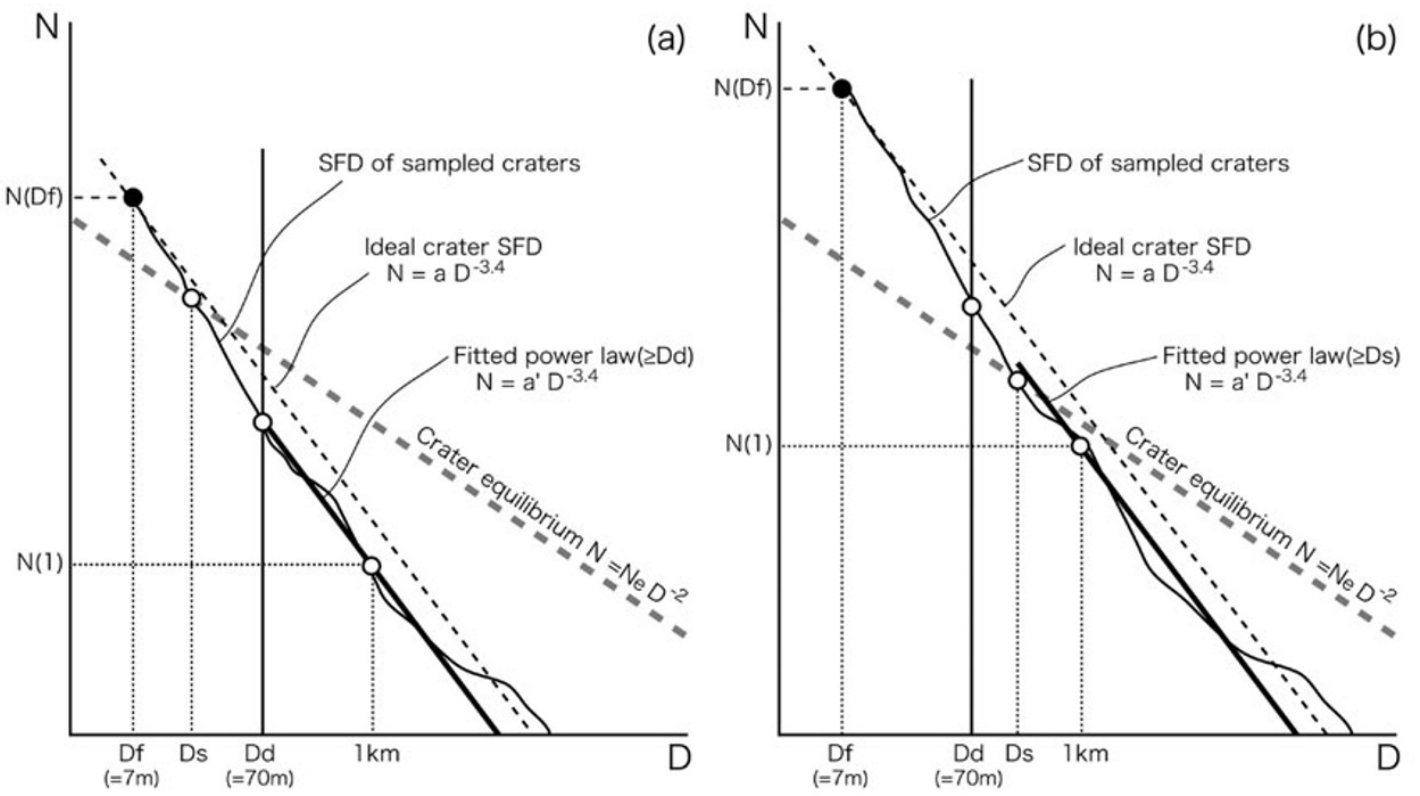

Fig. 1. Schematic representation of crater SFD measurement. Relative age is obtained by fitting the population SFD, $N(D)=\alpha D^{-3.4}$ to SFD of craters $\geq D_{m}$. (a) $D_{d} \geq D_{s}$. Craters $\geq D_{d}$ are used for SFD measurement (i.e., $D_{m}=D_{d}$ ). (b) $D_{d}<D_{s}$. We use $D_{m}=D_{s}$.

study, we assign $b=-3.4$ to the power index of crater population SFD. This value is well fitted for a medial size distribution of lunar sub-kilometer craters (e.g., Namiki and Honda, 2003).

We attempt to test geological units given various areas and ages. The ages are recognized as true ages for evaluating crater SFD fluctuation. For each unit, craters are randomly sampled by the time the number of craters larger than a crater size $D_{f}$ amounts to an ideal crater number calculated from the given area and age. To calculate the ideal number of craters, we use the size-frequency relation $N(D) \propto D^{-3.4}$ and Neukum's cratering chronology curve as follows,

$$
\begin{aligned}
N(D \geq 1 \mathrm{~km})= & 5.44 \times 10^{-14}\{\exp (6.93 \times t)-1\} \\
& +8.38 \times 10^{-4} t,
\end{aligned}
$$

where $t$ is the age in Gyr (Neukum, 1983; Neukum and Ivanov, 1994).

In our Monte Carlo simulation, the crater population SFD is used as the probability distribution for random sampling. Using a uniformly distributed pseudo-random number $0<$ $x<1$, a diameter of a sampled crater $D$ is given by the following expression,

$$
D=x^{-1 / b} D_{f}
$$

We use the linear congruential generators (Lehmer, 1951) for the random number generation. In our simulation, the random number generator has a period of $2^{48}$, which is sufficiently long for the purpose of this study.

The number at $D_{f}$ does not fluctuate because of the apriorism, but frequencies for larger craters are expected to fluctuate randomly around the ideal crater SFD. In order to correctly simulate the statistical fluctuation of the SFD, $D_{f}$ must be given a value much smaller than a minimum crater size $D_{m}$ used for crater SFD measurement. We set

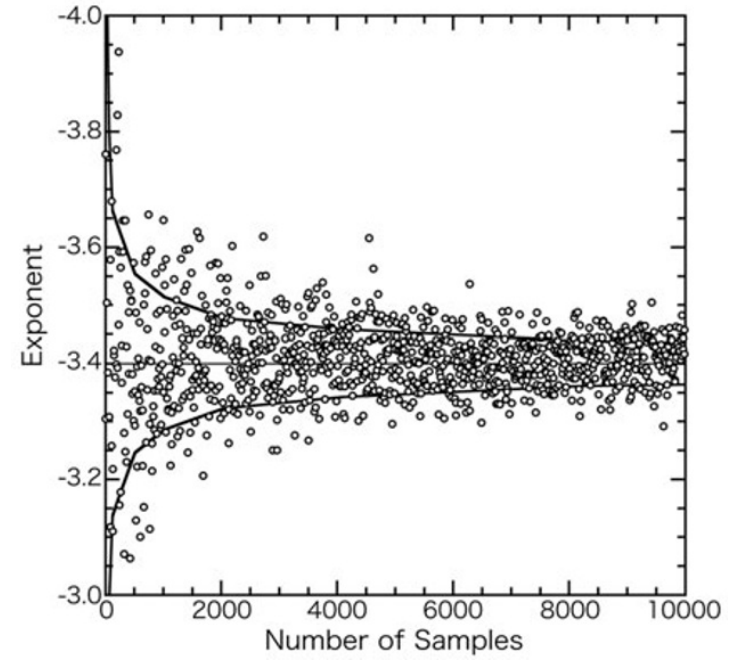

Fig. 2. Exponent of the power-law fitted to SFD for the sampled crater set as a function of the number of samples. The exponent assigned to the crater population SFD is -3.4. Solid lines denote one standard deviation.

$D_{f}=7 \mathrm{~m}$. As described below, the value is smaller than $0.1 D_{m}$. Therefore, the effect of the crater number fixed at $D_{f}=7 \mathrm{~m}$ is negligible; according to $N(D) \propto D^{-3.4}$, the number at $D_{f}$ is about 2500 times larger than that at $D_{m}$, so the fluctuation at $D_{f}$ is estimated to be less than $2 \%$ of that at $D_{m}$ using the standard deviation given by $\sqrt{N}$. Hereafter, we use only the crater density, and the spatial distribution of craters is excluded in our simulation.

\subsection{Measurement of SFD and age determination}

We use cumulative plots that give the number of craters larger than a certain diameter per unit area (e.g., Crater Analysis Techniques Working Group, 1979). The crater diameters given from Eq. (3) are binned before plotting. We divide an order of magnitude into 16 bins that have a 


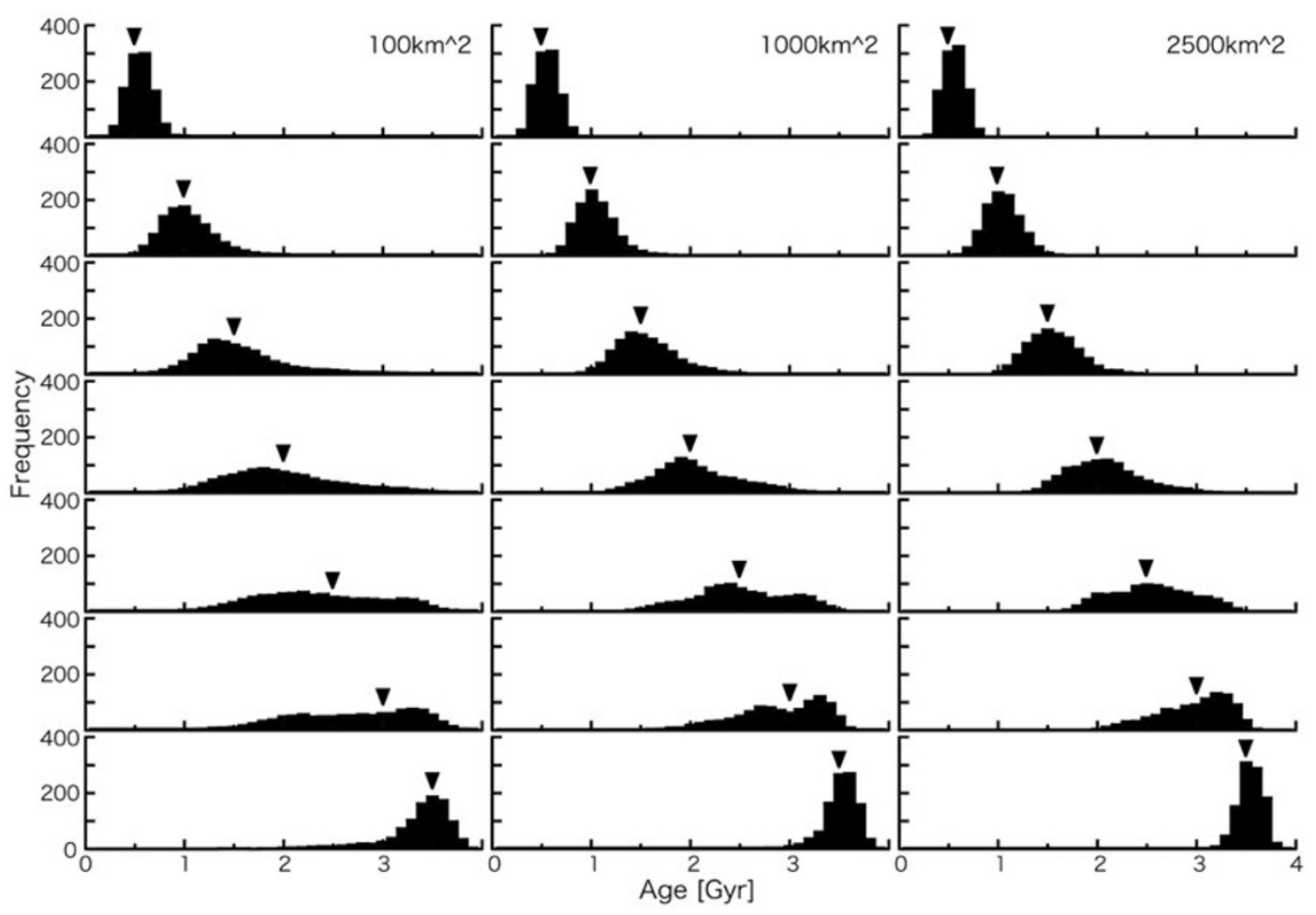

Fig. 3. Histogram of estimated ages for $N_{e}=0.015$. Filled inverse triangle indicates the true age of the test geological unit.

constant interval in the log plot.

We postulate that the minimum $D_{d}$ of detectable crater with the TC images is $70 \mathrm{~m}$ since the nominal resolution is about $10 \mathrm{~m}$. Therefore, we use craters $\geq 70 \mathrm{~m}$ for CSFD measurement. In the real situation, however, the quality of crater counts depends not only on the image resolution but also many other factors; e.g., the illumination conditions of images (Young, 1975; Honda et al., 2006) and the crater preservation state related to the geologic setting (e.g., Basilevsky, 1976). Although these factors may not be negligible, we exclude them to simplify the simulation. These effects must continue to be investigated using TC images and other high-resolution images obtained by future missions.

According to previous studies by impact experiments and simulations (e.g., Gault, 1970), crater saturation equilibrium occurs between $N(D=1)=0.015 \sim 0.15$ when the SFD exponent of saturated surfaces is close to -2 . In general, a number density of craters smaller than a few hundred meters in diameter on lunar maria is expected to reach the saturation level (e.g., Hartmann, 1984; Hartmann and Gaskel, 1997). The saturated craters are not representative of the distribution and must be excluded from crater SFD measurement. In our simulation, the saturation equilibrium is not reached since the spatial distribution of sampled craters is not considered. Therefore, we use just the crater density in order to judge whether craters are saturated or not. We judge the "saturation equilibrium condition" to be met when the crater density exceeds the following crater density,

$$
N(D)=N_{e} D^{-2}
$$

If the crater density at $D_{d}$ is saturated, only craters larger than the maximum size $D_{s}$ of saturated craters are used for crater SFD measurement. Figure 1 schematically represents the relations among $D_{d}, D_{s}$, and $D_{m}$. In this study, we investigate cases of $N_{e}=0.015,0.045$, and $0.075 \mathrm{~km}^{-2}$, corresponding to 1,3 , and $5 \%$ of the geometric saturation, respectively. (Gault (1970) found 5 to $7 \%$ as the preferred saturation level. However, a "fall-off" of SFD occurs at a lower crater density. For real geological units, data within this "fall-off" should be excluded from the analysis. Therefore we use the lower crater density as the saturation level.) In addition, a case in which the saturation equilibrium condition is not considered (i.e. $N_{e}=\infty$ ) is also studied for comparison.

The relative age can be obtained by fitting the standard size-frequency distribution that is estimated from the average of measured SFDs to the measured crater distribution. In our simulation, we use the crater population SFD, $N(D)=\alpha D^{-3.4}$ as the standard size distribution because we can precisely estimate the crater population SFD by averaging a large number of measured crater SFDs; Fig. 2 graphs the exponent of the power-law fitted to SFD of the sampled crater as a function of the number of samples. According to this, if we count more than 10,000 craters, we can estimate the exponent of the crater population SFD within an error of \pm 0.04 . Although this estimate may be slightly different from the actual error since the real lunar SFD may not follow a simple power law (e.g., Neukum et al., 1975a), we can expect that the error in the determination of the standard SFD is negligible.

The power-law $N(D)=\alpha D^{-3.4}$ is fitted to the SFD measured for craters $\geq D_{m}$ by the least square method in order to estimate $\alpha$ representing the relative age of a geologic unit. In a real situation, observed SFDs may be misshaped 
by various geologic factors, such as flooding, blanketing, secondary cratering, and volcanic craters (Neukum et al., 1975a). In such cases, a statistical verification of the fitting error, such as the $\chi^{2}$ test, is needed to judge whether the standard SFD can be fitted to the observed SFD or not. However, our simulation does not include the geological effects, so deviation of individual SFDs from the simple power law is not tested. Substituting the estimated $\alpha$ for $N(D \geq 1 \mathrm{~km})$ in Eq. (2), the absolute age $t$ of the unit is determined. We compare the estimated ages with the true age.

\section{Results and Discussion}

For each test unit, we produced 1000 crater sets by random sampling and age determination. Figure 3 displays the histogram of ages estimated for each test unit. As expected, the dispersion of estimated ages decreases with increasing area, that is, with an increased number of available craters. We can also see that the ages estimated for units of 2.0 to 3.0 Gyr are largely dispersed. This is due to the shape of the chronology curve that has low age resolution in this range.

Figure 4 compares the average of estimated ages and the true age of the test unit; the average of estimated age is consistent with the true age. However, the ages estimated for units of 2.5 to $3.5 \mathrm{Gyr}$ tend to be too young when there are not enough craters. This is because the cratering chronology curve has a steeper slope before 3.2 Gyr than after that (see Eq. (2), or Neukum and Ivanov, 1994, figure 16). For crater frequencies dispersed randomly around the crater frequency corresponding to $3.2 \mathrm{Gyr}$, ages of units with lower crater frequencies tend to be widely underestimated, while ages of units with higher frequency are slightly overestimated. As a consequence of the dissymmetry, the average age is estimated to be younger than the true age. However, this is insignificant when the dispersion of crater frequencies is not so large.

Figure 5 plots the standard deviation of estimated ages as a function of the true age of the test geological unit. It can be seen that age determination for Eratosthenian units (1.1 to $3.2 \mathrm{Gyr}$ ) is the least accurate. The standard deviations are $0.7 \mathrm{Gyr}$ for units of $100 \mathrm{~km}^{2}, 0.5 \mathrm{Gyr}$ for units of $1000 \mathrm{~km}^{2}$, and $0.4 \mathrm{Gyr}$ for units of $2500 \mathrm{~km}^{2}$. The maximum error amounts to over $20 \%$ and is comparable to the error due to cratering asymmetry (Morota et al., 2005, 2007). Previous studies (e.g., Greeley et al., 1993; Hiesinger et al., 2000, $2003,2006)$ used an age range calculated by $\pm \sqrt{N}$ as the error of age determination. The standard deviation obtained in this study is much larger than that, although it is difficult to simply compare results because of different image resolutions and areas of geological units.

Figure 6 displays the relationship between age and maximum size of saturated craters for each equilibrium level. This maximum size of saturated craters is obtained by calculating a node between the SFD of a saturated crater (Eq. (3)) and an ideal SFD expected for a given age. From Fig. 6, ages when a $70 \mathrm{~m}$-diameter crater is saturated are expected to be $0.43 \mathrm{Gyr}$ for $N_{e}=0.015 \mathrm{~km}^{-2}, 1.29 \mathrm{Gyr}$ for $N_{e}=0.045 \mathrm{~km}^{-2}$, and $2.16 \mathrm{Gyr}$ for $N_{e}=0.075 \mathrm{~km}^{-2}$, and are indicated as triangles in Fig. 5. In Fig. 5, the ages are consistent with ages deviating from plots for $N_{e}=\infty$.
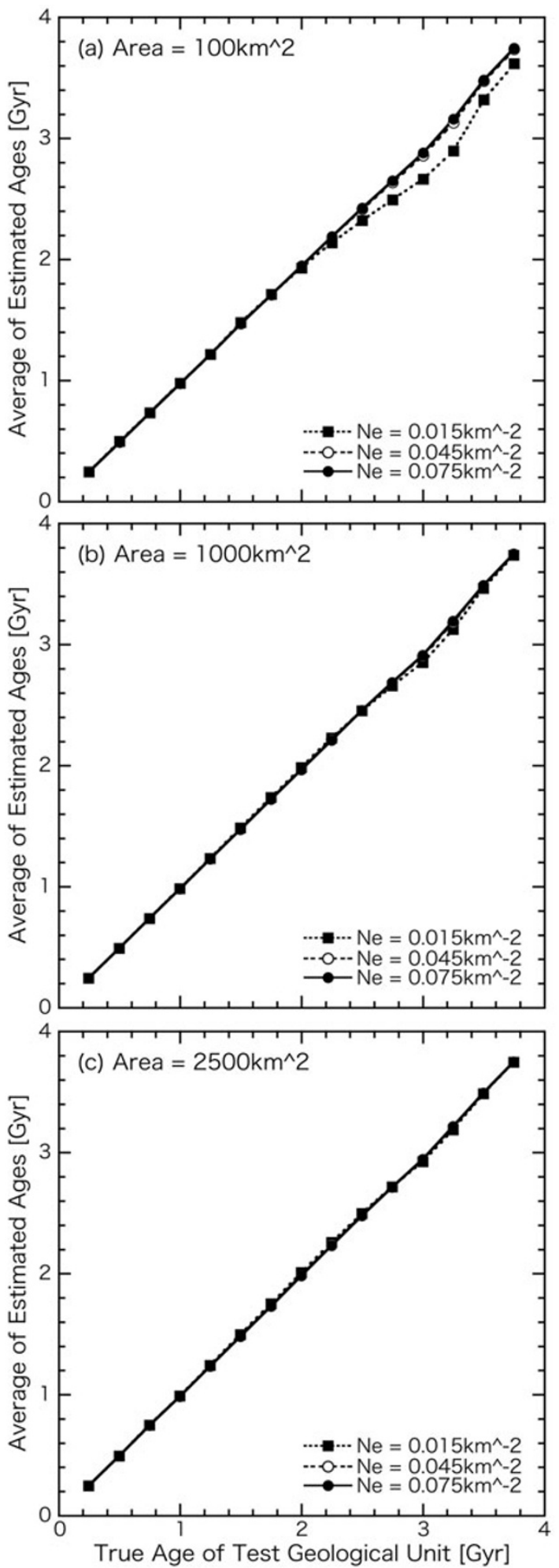

Fig. 4. Comparison of the average of estimated ages and the true age of the test geologic unit for 100,1000 , and $2500 \mathrm{~km}^{2}$.

Figure 6 indicates that $70 \mathrm{~m}$ craters in Copernican units have not been saturated yet. Therefore, we should be able to continue to improve dating certainty for Copernican units by acquiring higher-resolution images on future missions. In contrast, the Eratosthenian units have been saturated by craters $<70 \mathrm{~m}$. In order to improve the accuracy of Eratosthenian age, we need additional information, such as 

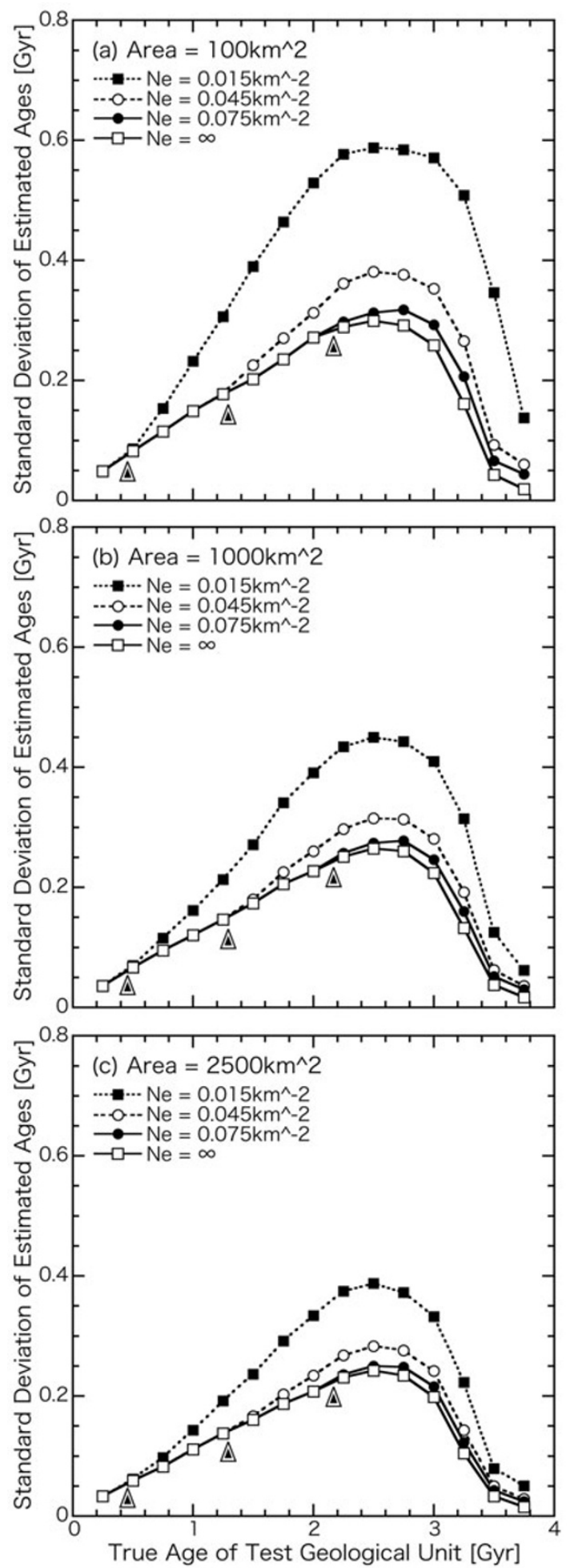

Fig. 5. Standard deviation of estimated ages as a function of the true age of the test geological unit. Triangle indicates the age when a $70 \mathrm{~m}$-diameter crater is saturated for a given equilibrium level (see Fig. 6).

the degradation of crater morphology (e.g., Soderblom and Lebofsky, 1972).

We suggest a new approach for investigating the lunar primary/secondary cratering proportion. This study estimated the fluctuation of crater frequency based on the single crater population. A comparison of the results with the lunar cratering record may yield new insight onto the lunar

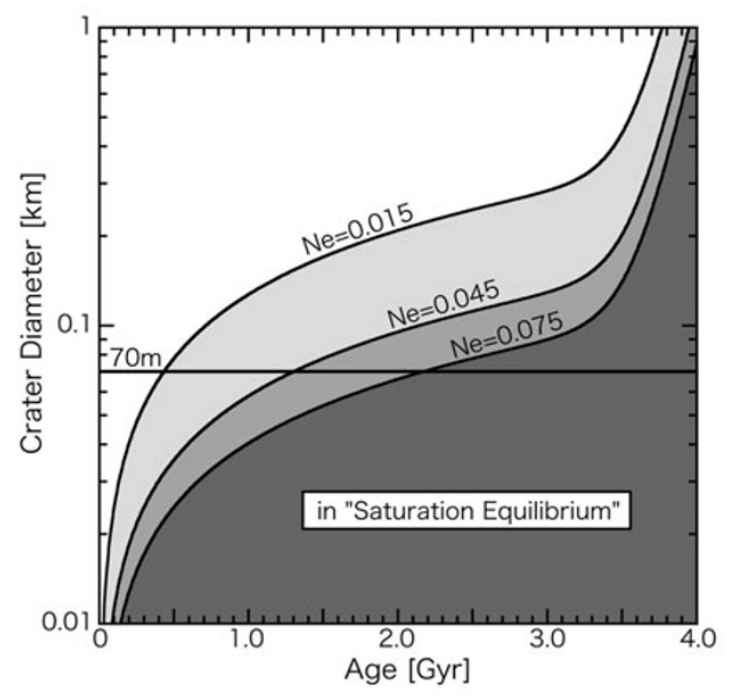

Fig. 6. Relationship between age and maximum size of saturated craters for each equilibrium level. The maximum size of saturated craters is obtained by calculating a node between the SFD of a saturated crater (Eq. (3)) and the ideal SFD expected for a given age. The ideal SFD can also be calculated from the cratering chronology (Eq. (2)) and the population SFD.

crater population. For example, a large number of subunits derived from a lunar geological unit could be individually dated by crater counting. The ages estimated for individual subunits must be dispersed even if they are from the same geological unit. If the dispersion of ages is significantly large compared with our simulation results, the SFD of lunar small craters may be complicated both by statistical fluctuation and by secondary craters. Conversely, if the observed dispersion is close to our estimate, this means that most small craters are primary craters, or that primary and secondary populations have the same SFD. This approach requires a large enough homogenous unit. If such units exist, this will be evaluated with TC image data.

In addition to the possible secondary crater contamination, the problem lies in defining units by albedo and/or spectral characteristics. In this case, the possible nonuniform distribution of craters over the area would generate errors exceeding our estimates. However, we expect that we can avoid the problem of accurate unit definition by the Multiband Imager (Ohtake et al., 2000, 2007) and Spectral Profiler (Matsunaga et al., 2000, 2002) installed on SELENE (KAGUYA) with the statistic test for analysis of spatial distribution of craters (Kreslavsky, 2007).

\section{Conclusions}

Assuming that small craters can be employed for age determination, we evaluated the statistical fluctuation of crater frequency and its effect on age determination by random sampling simulation. The results demonstrate that age determination for Eratosthenian units is least accurate, and its maximum error amounts to over $20 \%$. However, at present, the uncertainty of this dating method exceeds $20 \%$, owing to the probable contamination by secondary craters.

In order to improve the age-determination accuracy of lunar geological units with TC images, additional infor- 
mation about the degradation of crater morphology (e.g., Soderblom and Lebofsky, 1972) is needed. We will obtain a lunar Digital Terrain Model (DTM) with a spatial resolution of $10 \mathrm{~m}$ by TC stereo imaging (Haruyama et al., 2006b; Yokota et al., 2007). The height resolution of the DTM is expected to exceed $15 \mathrm{~m}$ from results of the preliminary test (Honda et al., 2007). Using crater frequency and crater degradation measured with TC images and DTM complementarily, we will try to determine the ages of geological units on the entire Moon and infer the lunar geological history.

Acknowledgments. We are grateful to B. A. Ivanov and an anonymous reviewer for thoughtful and constructive comments in their review of this paper. We also thank J. Oberst for his helpful comments.

\section{References}

Baldwin, R. B., Relative and absolute ages of individual craters and the rate of infalls on the moon in the post-imbrium period, Icarus, 61, 6391, 1985.

Basilevsky, A. T., On the evolution rate of small lunar craters, Proc. Lunar Sci. Conf. 7th, 1005-1020, 1976.

Chapman, C. R. and R. R. Haefner, A critique of methods for analysis of the diameter-frequency relation for craters with special application to the moon, J. Geophys. Res., 72, 549-557, 1967.

Crater Analysis Techniques Working Group, Standard techniques for presentation and analysis of crater size-frequency data, Icarus, 37, 467474, 1979.

Dundas, C. M. and A. S. McEwen, Rays and secondary craters of Tycho, Icarus, 186, 31-40, 2006.

Gault, D. E., Saturation and equilibrium conditions for impact cratering on the lunar surface, Radio Sci., 5, 273-291, 1970.

Greeley, R., 12 colleagues, Galileo imaging observations of lunar maria and related deposites, J. Geophys. Res., 98, 17183-17205, 1993.

Hartmann, W. K., Lunar cratering chronology, Icarus, 13, 299-301, 1970.

Hartmann, W. K., Paleocratering of the Moon: Review of post-Apollo data, Astrophys. Space Sci., 17, 48-64, 1972.

Hartmann, W. K., Does crater "saturation equilibrium" occur in the Solar system, Icarus, 60, 56-74, 1984.

Hartmann, W. K. and R. W. Gaskel, Planetary cratering 2: Studies of saturation equilibrium, Meteorit. Planet. Sci, 32, 109-121, 1997.

Haruyama, J., H. Otake, M. Ohtake, A. Shiraishi, N. Hirata, and T. Matsunaga, LISM (Lunar Imager/SpectroMeter) mission for SELENE project, Lunar Planet. Sci. XXXI, Abstract \#1317, 2000.

Haruyama, J., M. Ohtake, N. Hirata, R. Nakamura, and T. Matsunaga, Expected performance of Lunar Imager/SpectroMeter on SELENE, Lunar Planet. Sci. XXXIV, Abstract \#1565, 2003.

Haruyama, J., M. Ohtake, T. Matsunaga, and LISM working group, Global high-resolution stereo mapping of the Moon with the SELENE Terrain Camera, Adv. Geosci., 3, 101-108, 2006a.

Haruyama, J., M. Ohtake, T. Matsunaga, T. Morota, A. M. Yoshizawa, and LISM working group, Planned Digital Terrain Model products from SELENE Terrain Camera data, Lunar Planet. Sci. XXXVII, Abstract \#1132, 2006b.

Haruyama, J., T. Matsunaga, M. Ohtake, T. Morota, C. Honda, Y. Yokota, M. Torii, Y. Ogawa, and the LISM Working Group, Global lunar-surface mapping experiment using the Lunar Imager/Spectrometer on SELENE, Earth Planets Space, 60, this issue, 243-255, 2008.

Hiesinger, H., R. Jaumann, G. Neukum, and J. W. Head III, Age of mare basalts on the lunar nearside, J. Geophys. Res., 105, 29239-29275, 2000.

Hiesinger, H., J. W. Head III, U. Wolf, R. Jaumann, and G. Neukum, Ages and stratigraphy of mare basalts in Oceanus Procellarum, Mare Nubium, Mare Cognitum, and Mare Insularum, J. Geophys. Res., 108, doi:10.1029/2002JE001985, 2003.

Hiesinger, H., J. W. Head III, U. Wolf, R. Jaumann, and G. Neukum, New ages for basalts in Mare Fecunditatis based on crater size-frequency measurements, Lunar Planet. Sci. XXXVII, Abstract \#1151, 2006.

Hirata, N. and A. M. Nakamura, Secondary craters of Tycho: sizefrequency distributions and estimated fragment size-velocity relationships, J. Geophys. Res., 111, E03005, doi:10.1029/2005JE002484,
2006.

Honda, C., T. Morota, and J. Haruyama, A determination of surface ages of small geologic units on the terrestrial planets and satellites, 36th COSPAR Scientific Assembly, Abstract \#2783, 2006.

Honda, C., T. Morota, Y. Yokota, J. Haruyama, M. Ohtake, T. Matsunaga, Y. Ogawa, H. Demura, N. Hirata, A. Iwasaki, S. Kodama, S. Hara, K. Hioki, and LISM working group, Preliminary test of accuracy of Digital Terrain Model derived from SELENE/LISM/TC data, Lunar Planet. Sci. XXXVIII, Abstract \#1899, 2007.

Ivanov, B. A., Earth/Moon impact rate comparison: searching constraints for lunar secondary/primary cratering proportion, Icarus, 183, 504-507, 2006.

Kreslavsky, M. A., Statistical characterization of spatial distribution of impact craters: implications to present-day cratering rate on Mars, 7 th Inter. Conf. Mars, Abstract \#3325, 2007.

Lehmer, D. H., Mathematical methods in large-scale computing units, In Proceedings of 2nd Symposium on Large-Scale Digital Calculating Machinery, Harvard Univ. Press, pp. 141-146, 1951.

Matsunaga, T., M. Ohtake, Y. Hirahara, and J. Haruyama, Development of a visible and near infrared spectrometer for Selenological and Engineering Explorer (SELENE), Proc. SPIE, 4151, 32-39, 2000.

Matsunaga, T., M. Ohtake, and T. Sugihara, Environmental test results and their implications to the performance of SELENE Spectral Profiler, Proc. 23rd Inter. Symp. Space Techn. Sci., 1922-1927, 2002.

McEwen, A. S. and E. B. Bierhaus, The importance of secondary cratering to age constraints on planetary surfaces, Ann. Rev. Earth Planet. Sci., 34, 535-567, 2006.

McEwen, A. S., B. S. Preblich, E. P. Turtle, N. A. Artemieva, M. P. Golombek, M. Hurst, R. L. Kirk, D. M. Burr, and P. R. Christensen, The rayed crater Zunil and interpretations of small impact craters on Mars, Icarus, 176, 351-381, 2005.

Morota, T., T. Ukai, and M. Furumoto, Influence of the asymmetrical cratering rate on the lunar cratering chronology, Icarus, 173, 322-324, 2005.

Morota, T., J. Haruyama, C. Honda, Y. Yokota, M. Ohtake, and M. Furumoto, Lunar apex-antapex cratering asymmetry as an impactor recorder in the Earth-Moon system, Adv. Space Res., 2007 (in press).

Namiki, N. and C. Honda, Testing hypotheses for the origin of steep slope of lunar size-frequency distribution for small craters, Earth Planets Space, 55, 39-51, 2003.

Neukum, G., Meteoritenbombardement und Datierung planetarer Oberflachen, Habilitation Dissertation for Faculty Membership, Ludwig Maximilianis Univ., Munich, 1983.

Neukum, G. and B. A. Ivanov, Cratering size distributions and impactprobabilities on Earth from lunar, terrestrial planet, and asteroid cratering data in Hazards Due to Comet and Asteroids, edited by T. Gehrels, pp. 359-416, Univ. of Arizona Press, Tucson, 1994.

Neukum, G. and B. Konig, Dating of individual lunar craters, Proc. Lunar Sci. Conf. 7th, 2867-2881, 1976.

Neukum, G., B. Konig, and J. Arkani-Hamed, A study of lunar impact crater size-distributions, The Moon, 12, 201-229, 1975a.

Neukum, G., B. Konig, H. Fechtig, and D. Storzer, Cratering in the earthmoon system: Consequences for age determination by crater counting, Proc. Lunar Sci. Conf. 6th, 2597-2620, 1975b.

Ohtake, M., J. Haruyama, H. Otake, and T. Matsunaga, LISM mission for the SELENE project, Proc. 4th Int. Conf. On the Exploration and Utilisation of the Moon, 203-206, 2000.

Ohtake, M., J. Haruyama, T. Matsunaga, S. Kodama, T. Morota, and Y Yokota, Scientific objectives and specification of the SELENE Multiband Imager, Adv. Space Res., 2007 (in press).

Soderblom, L. A. and L. A. Lebofsky, Technique for rapid determination of relative ages of lunar areas from orbital photograpy, J. Geophys. Res., 77, 279-296, 1972.

Wilhelms, D. E., Geologic history of the Moon, U.S. Geol. Surv. Prof. Pap., 1348, 302 pp, 1987.

Yokota, Y., J. Haruyama, M. Ohtake, T. Matsunaga, C. Honda, T. Morota, H. Demura, N. Hirata, and LISM working group, Kilometer scale roughness analysis of lunar digital terrain model, Lunar Planet. Sci. XXXVIII, Abstract \#2430, 2007.

Young, R. A., Mare crater size-frequency distributions: implications for relative surface ages and regolith, Proc. Lunar Sci. Conf. 6th, 2645$2662,1975$.

T. Morota (e-mail: morota.tomokatsu@jaxa.jp), J. Haruyama, C. Honda, Y. Yokota, M. Ohtake, Y. Ogawa, and T. Matsunaga 